\title{
La civilité électorale : vote et forclusion de la violence en France (Partie 2)
}

Olivier Ihl

\section{(2) OpenEdition}

\section{Journals}

Édition électronique

URL : http://journals.openedition.org/conflits/640

DOI : $10.4000 /$ conflits. 640

ISSN : $1777-5345$

Éditeur :

CCLS - Centre d'études sur les conflits lilberté et sécurité, L'Harmattan

Édition imprimée

Date de publication : 15 mai 1993

ISSN : 1157-996X

\section{Référence électronique}

Olivier Ihl, «La civilité électorale : vote et forclusion de la violence en France (Partie 2) », Cultures \&

Conflits [En ligne], 09-10 | printemps-été 1993, mis en ligne le 02 mars 2005, consulté le 30 mars 2021.

URL : http://journals.openedition.org/conflits/640 ; DOI : https://doi.org/10.4000/conflits.640

Ce document a été généré automatiquement le 30 mars 2021.

Creative Commons License 


\title{
La civilité électorale : vote et forclusion de la violence en France (Partie 2)
}

\author{
Olivier Ihl
}

\section{L'ordre du vote contesté}

On l'a vu: construit spatialement et normativement, le vote instaure un ordre symbolique qui impose une éradication complète des expériences de violence. Aussi, dans l'imaginaire démocratique, l'électeur-citoyen a-t-il pour figure symétriquement inverse l'insurgé : celui, dont la conduite dans l'espace public opère une sorte d'inversion du comportement idéal que les manuels scolaires assignent au futur citoyen. Cependant, cet ordre aux multiples figures n'a pas annulé immédiatement les passions et les violences politiques. L'usage du bulletin de vote n'a pas empêché des électeurs de continuer à prendre leur fusil ou leur fourche pour défendre leurs intérêts menacés. Il suffit pour s'en convaincre de rappeler que l'année symbole du suffrage universel - 1848 - est aussi celle qui ouvre l'une des périodes les plus violentes de l'histoire française. Les recherches de Charles Tilly révèlent une contestation persistante de la morale sociale née de la pratique universelle du vote. Etablissant la courbe des violences collectives survenues en France de 1830 à 1960, le sociologue américain montre que, globalement, la participation aux actions violentes augmente entre le XIXe et le XXe siècle, passant d'une moyenne de 79 manifestions pour 100000 habitants à 105. Au delà de cette évolution quantitative, il importe de noter que la répartition géographique des violences recensées est très inégale. Les départements ruraux sont souvent le théâtre d'agressions et de brutalités collectives. La présence d'un centre urbain en expansion favorise aussi le développement de certaines formes de violence. C'est dire que l'affirmation d'une civilité politique - indissociable de l'accoutumance au vote - n'agit pas avec la même force sur l'ensemble du territoire national. Si certains départements sont précocement pacifiés d'autres restent 
durablement troublés ${ }^{1}$. C'est dire également que l'entreprise d'illégitimation de la violence en oeuvre dans le vote rencontra de fortes résistances. La disqualification des moyens d'action politique traditionnels ne s'est opérée ni de façon linéaire, ni de manière docile. S'ils permettent de nuancer l'optimisme des promoteurs du vote comme mode exclusif de désignation politique, les travaux de Tilly permettent, en outre, d'apprécier l'évolution des formes d'expression de la violence. Si la culture démocratique ne fait pas disparaître la violence instrumentale ou colérique ${ }^{2}$, elle tend cependant à modifier son répertoire d'action. Au début du XIXe siècle, les actions collectives de violence prennent souvent la forme de mouvements spontanés, faiblement organisés (foules, émeutes agraires, résistances au fisc, prises de grain...). En revanche, dans le courant du XIXe siècle et du XXè siècle, ces actions tendent de plus en plus à s'organiser sur une base professionnelle et supra-locale. De même, de telles actions font l'objet d'un encadrement de plus en plus manifeste (grèves, manifestations...) ${ }^{3}$. C'est peut-être dans cette évolution qualitative qu'il faut voir le principal effet de l'acculturation civique. Il ne saurait être question, dans les limites de cet article, de répondre de façon définitive et complète à l'hypothèse. On peut cependant tenter d'explorer à partir d'un terrain d'enquête circonscrit, les nouvelles relations qu'entretiennent vote et violence à la fin du XIXe siècle. La lecture des documents d'archives ou de presse disponibles fait, en effet, apparaître un objet cohérent : l'espace du vote et son voisinage comme lieu d'expérimentation d'un nouvel équilibre entre le vote et la violence politique.

\section{Physionomie (s) d'une journée électorale.}

Si on quitte l'histoire des textes (juridiques ou pédagogiques) pour passer à celle des comportements politiques effectifs, on s'aperçoit que les réticences à investir les attributs du rôle de citoyen sont nombreuses. Par là se trouvent mis en lumière les limites de l'incidence des dispositions protégeant le vote. Des dizaines d'années après l'épisode de 1848 , les autorités préfectorales craignent encore que les opérations électorales ne soient perturbées par des manifestations qui remettraient en cause l'ordre démocratique. C'est ainsi que, lors du scrutin législatif du 27 avril 1902, la préfecture de Police de Paris a mobilisé plus de cent quarante gendarmes et près de neuf cents fantassins et cavaliers de la Garde Républicaine. Symboliquement, le poste militaire de l'Elysée a également été doublét. Loin d'être seulement le reflet de l'agitation nationaliste, ce déploiement de force se retrouve chaque jour d'élection. Une réalité qui contraste avec la représentation que donne la presse parisienne $\mathrm{du}$ déroulement électoral. Les principaux organes de presse insistent toujours, eux, sur la dimension paisible et sereine du vote. Description standard, celle du journal Le Téléphone en 1902 : "Rarement un jour d'élections fut plus calme que celui-ci. Dans les rues où se trouvent des bureaux de section de vote des 1er et $2 \mathrm{e}$ arrondissements et dans les rues avoisinantes, l'animation est nulle, comme un matin de dimanche ordinaire. La pluie qui ne cesse de tomber a sans doute rafraîchi les cerveaux exaltés par la lecture des manifestes multicolores qui fatiguent les yeux un peu partout, ou bien les bons électeurs jouissent tranquillement dans leur lit du repos hebdomadaire et se réservent pour l'après-midi la promenade au vote. (...) Même calme à la mairie du 17e arrondissement..." 5. Les mêmes idiolectes reviennent constamment sous la plume 
des journalistes : la "sagesse" des électeurs qui savent "raisonner leur comportement", "limiter leur passion" à l'accomplissement d'un devoir civique. Ces récits contribuent à consolider l'un des stéréotypes les plus utilisés des discours démocratiques : celui qui fige l'acte du vote dans des attendus de sérénité, de clairvoyance et de dignité. En contant l'épisode électoral selon les canons de l'éthique démocratique, la presse impose progressivement une image convenue du déroulement d'une journée électorale. S'y déploient des personnages fonctionnels (les distributeurs de bulletin, les colleurs d'affiche, les scrutateurs...) et des situations archétypales (le vote du président de la République, celui des pensionnaires de l'hôtel des Invalides, celui des ecclésiastiques...). $\mathrm{Au}$ total, l'effet de ces lieux communs est de convaincre du caractère à la fois routinier et exemplaire de l'acte du vote. L'un des points de passage obligés des récits électoraux consiste à solenniser les opérations électorales. Le bon déroulement de la procédure est censé témoigner de la réussite de l'entreprise de pacification des moeurs politiques. La tranquillité électorale est notamment perçue comme un indicateur de la vigueur des attendus normatifs du vote : "Cette journée d'élections se passa dans un calme admirable. (...) Les électeurs ont rempli leur devoir avec la dignité qui sied à des hommes libres et conscients"6. Reste à expliquer la distorsion entre l'importance des forces de l'ordre mobilisées pour protéger les opérations électorales et le calme dans lequel ces dernières semblent se dérouler. Si la mobilisation de la violence légitime renvoie pour partie à une politique de prévention, elle reflète également une inégale pacification de l'espace électoral. La résolution du paradoxe suppose, en fait, une attention renouvelée aux lieux et temporalités de l'acte du vote. La forclusion de la violence s'exerce de manière variable. Si la salle de vote est rarement affectée par des violences collectives, l'intensité des perturbations tend à augmenter au fur et à mesure que l'on s'éloigne de cet espace sacralisé. La turbulence qui caractérise l'espace de la rue, en ces années où la fête électorale garde toutes ses lettres de noblesse, contraste avec le calme qui règne à l'intérieur du bureau de vote. Une citoyenneté rebelle, plus bruyante, se fait alors jour hors du cercle des formes euphémisées d'activité politique ou en tout cas sur ses marges ${ }^{7}$. Dans la salle même du vote, les écarts aux règles de neutralisation de la violence sont rares. La sacralisation dont bénéficie cet espace, les pouvoirs de police détenus par le président de bureau, la docilité imposée par les technologies du vote contribuent à endiguer le surgissement de la violence. Certains électeurs refusent quelques fois cette posture citoyenne. Sans prétendre établir une typologie de leurs manifestations, on peut d'ores et déjà dégager deux types essentiels de litiges. Le premier rassemble les incidents dont l'origine est la présence dans la salle du vote d'une personne qui, de par ses qualités ou la place qu'elle occupe dans la société, apparaît comme contraire à la solennité politique du lieu. La force de la mémoire anticléricale dans certains quartiers parisiens entraîne ainsi de nombreux incidents lors de la présence d'ecclésiastiques dans les sections de vote. Lors des élections du 11 mai 1902, le bureau de vote de la cité Voltaire dans le 11ème arrondissement fut agité de bruits et de bousculades lors de l'arrivée dans la salle d'un prêtre en soutane. "Parmi les gens présents les uns, connaissant cet abbé qui serait un ancien capitaine d'Artillerie, ont pris sa défense, tandis que d'autres se sont mis à l'attaquer..." 8. Dans le 20ème arrondissement, c'est la présence d'"un idiot" qui perturbe l'ordre du vote : "Un sieur Soudieu Emile, porteur aux Halles, domicilié rue de Fontarabie, 25, qui est idiot s'est présenté quelques instants avant la fermeture du scrutin pour voter. Les électeurs présents ont protesté et ont empêché cet inconscient de déposer son bulletin dans l'urne. Sa carte d'électeur a été conservée par le président 
et l'affaire inscrite au procès-verbal de la section"'. De tels incidents renseignent sur le code normatif sous-jacent au scrutin. Ils dessinent en négatif les frontières de la décence civique : en refusant d'adhérer au principe de la souveraineté populaire, en symbolisant le magistère d'une tutelle réprouvée, de nombreux prêtres se virent exclus d'une pratique civique pourtant ouverte à tous. De même faute de comprendre l'enjeu du scrutin, des personnes démunies des signes de la respectabilité citoyenne se virent refuser l'accès à l'urne. Ce qui explique les troubles provoqués par la présence d'un électeur en état d'ébriété dans la salle du vote. L'intervention des forces de l'ordre pour expulser ces "citoyens indignes" renforce alors le désordre constaté. Un autre cas de figure se rencontre fréquemment. Il s'agit du tumulte que provoque la présence dans la salle du vote d'un candidat impopulaire. Bien évidemment, toutes les luttes politiques ne débouchent pas sur des rixes collectives. La plupart du temps, c'est la séquence du dépouillement et la proximité immédiate de la salle du vote qui cristallisent les échauffourées. La salle du vote se trouve fréquemment perturbée par un second type de violence : celle qu'occasionnent les frustrations nés des entraves apportées aux opérations électorales. Ayant intériorisé la dimension matérielle du vote (lieu, horaires, rythme...), les électeurs peuvent se montrer décontenancés par les modifications introduites dans l'activité routinière du vote. Face à des changements soudains ou une lenteur excessive, ils peuvent réagir par de brusques sursauts de colère : "A $2 \mathrm{~h} 30 \mathrm{du}$ soir, à la section rue Boileau, les électeurs trouvant la distribution des cartes d'électeurs trop lente ont protesté et renversé tables et bancs. Le président de section a requis par écrit, les agents qui ont rétabli l'ordre dans la salle de vote puis sont retirés"10. L'imprévisibilité, même passagère, provoquée par les dérèglements $d u$ processus électoral laisse la voie ouverte à tous les débordements possibles. Si la majorité des citoyens parvient à conserver son calme, certains laissent libre cours à leur irritation. Les manifestations de colère traduisent alors comme une sorte de comportement régressif face au vote. Preuve s'il en est que les usages attestés du lieu exercent une contrainte non négligeable au point d'entraîner des gestes de dépit ou de colère lorsqu'un imprévu bouleverse l'équilibre des comportements prescrits. Il reste que les troubles surgis à l'extérieur de la salle continuent d'être à la fois plus fréquents et plus violents. Reflet des dispositions normatives qui trament l'accès au bureau de vote, la topographie de la violence marque les limites civiques du site électoral : la place publique, la cour de l'école, les abords immédiats du bureau, l'entrée du bourg... De façon générale, la rue permet de prolonger une compétition électorale que le vote aurait le tort d'euphémiser à l'excès. Il autorise la manifestation d'une citoyenneté rebelle, une citoyenneté opposée à l'ordre pacifié du vote et, surtout, à son "abstraction" jugée incapable d'épuiser l'intensité des engagements militants. Aussi, c'est en parcourant la ville, après la proclamation des résultats, que la plupart réinvestissent les luttes électorales de la part de violence que le vote évacue. La fête électorale, avec son lot de bravades et de chants partisans, vient largement "racheter" les déconvenues et les frustrations nées d'une procédure à la fois atomisée et impitoyable pour les minorités activistes. Aux abords des bureaux de vote, socialistes, anarchistes et nationalistes représentent les trois groupes politiques les plus souvent impliqués dans ces démêlés électoraux. Exemple parmi d'autres : à Paris, lors des élections législatives d'avril et mai 1902, le renouveau nationaliste provoqua de nombreuses bagarres dès la nuit tombée. C'est ainsi que, dans la soirée du 26 avril 1902, de graves rixes éclatèrent en plusieurs points de la capitale. Le motif déclaré en fut des "bandes d'individus (...) qui collaient sur des affiches électorales des imprimés"11 
portant les mentions "Electeurs! Attention! Ministériel veut dire Dreyfusard \& Antipatriote" ou encore "Candidat des juifs". A la violence des slogans s'ajoute une violence matérielle - l'usage des armes "blanches" est très répandu - sur laquelle les rapports de police apportent une documentation aussi minutieuse qu'abondante : "Ces trois derniers (il s'agit des trois personnes frappées pour avoir manifesté leur réprobation face à de tels procédés) qui étaient blessés ont été conduits à l'hôpital StLouis où ils ont été pansés. Girard, portait une forte blessure à la joue gauche et un coup de couteau à l'épaule du même côté. Renaud, une forte blessure saignante au dessus de l'oeil gauche où était resté un fragment de canne de dix centimètres de longueur environ. Francillard d'une blessure saignante à la main gauche et de plusieurs contusions à la tête..." ${ }^{12}$. Les multiples échauffourées qui ponctuent les soirées électorales attestent l'existence d'une violence électorale persistante ${ }^{13}$. Reléguée aux marges de l'espace électoral, cette violence épargne globalement l'intérieur du bureau de vote. Elle continue, en revanche, à occuper le domaine plus général de la rue. $\mathrm{Si}$, dans cette scénographie de la violence, la bagarre avec des adversaires politiques occupe une place non négligeable, d'autres formules existent : l'organisation d'un "désordre" devant le domicile de certains candidats ou encore la mise en place d'un défilé d'intimidation dans les rues proches de la salle du vote. Dans certains cas, l'occupation de l'espace public peut renvoyer à des formes de violence plus immatérielle. La violence symbolique se substitue alors à la violence physique mais sans en changer la fonctionnalité déclarée. C'est ainsi que lors des élections municipales de 1904, un millier de personnes ont parcouru les rues du 18e arrondissement avec des lampions acclamant l'élection de "leur" candidat. Le récit du fonctionnaire de la police municipal précise le but du cortège : "Ils ont promené une soutane qu'ils voulaient brûler sur la place. J'ai fait saisir la soutane. Le Grandais, monté sur une table devant le 74 de la rue du Mont-Cenis se mit à prononcer un discours devant 1200 assistants qui se proposent de le porter en triomphe..." ${ }^{14}$. La fréquence de ces épisodes festifs renseigne sur la marge de déviance acceptée le soir de l'élection. Mais, qu'on ne s'y méprenne pas : si la tolérance qui affecte cette partie de l'espace public est plus grande que celle autorisée dans le bureau de vote, elle ne saurait cependant être infinie. Les attroupements et défilés électoraux restent puissamment encadrés. Ils font l'objet d'une "gestion" policière incessante. Ainsi, les agents de police tentent toujours de négocier avec les manifestants la mise en scène choisie pour investir la rue. Et s'ils ne peuvent empêcher le déroulement du cortège lui-même, ils réussissent souvent à en infléchir les conditions concrètes de déploiement. A l'extérieur du bureau de vote, pas plus que dans l'espace de la salle elle-même, il n'est de place pour la "spontanéité" des inclinations politiques, signe que le vote est, par dessus tout, affaire de discipline et de civilité. Lieu façonné par une histoire sociale, culturelle et politique, le bureau électoral est une enceinte où se forment les identités personnelles, s'organise l'attachement aux valeurs démocratiques, s'éprouve l'appartenance à la communauté nationale. Espace concédé à des rassemblements éphémères, la salle de vote instaure une séparation protectrice entre un intérieur, domaine du recueillement, de la conscience et de l'intimité, et un extérieur abandonné aux affrontements, aux pressions et à la violence. Promis à des délibérations solitaires, ce théâtre de la subjectivité et du secret n'est pourtant qu'intermittent. Aménagé pour la circonstance dans des salles de mairie ou des écoles publiques, il s'institue à partir de signes provisoires et de technologies passagères dont le sort, une fois le scrutin passé, est de finir remisés au fond d'un hangar ou d'un placard. Les significations incorporées à ce site éphémère dépassent la 
seule gestuelle du vote. Elles concernent toute une série de valeurs morales qui rejaillissent au travers de conduites, infléchissent des attitudes, transforment les formes traditionnelles de rapport à soi. C'est en ce sens que l'on peut dire que l'emprise exercée par le bureau de vote sur les comportements agit en fait par astreinte normative. L'opposition paradigmatique entre le vote et la violence ne demeure pas une simple revendication éthique. Elle se traduit par une configuration précise des salles de vote, des procédures et des technologies qui les encadrent ou les animent. C'est l'un des intérêts majeurs de l'étude des conditions de spatialisation de l'acte du vote que de le montrer. Les répertoires du vote et de la violence se constituent sur des scènes séparées. Mais, loin de rester étrangers l'un à l'autre comme deux contraires irréconciliables, ils interagissent continuellement l'un sur l'autre. Rien d'étonnant à cela : la pratique du vote s'institue dans l'opposition dialectique aux actions traditionnelles de violence communautaire ou révolutionnaire. Son expérience périodique joue, dès lors, comme un rappel à l'ordre implicite. Elle se définit au travers d'attendus et de savoir-faire dont la logique est de dispenser de tout recours aux moyens de force. Réciproquement, la geste émeutière ou vindicative se constitue désormais par contraste avec les procédures électorales : de cette mise en relation, découle un renforcement de l'illégitimité morale et politique de ce type d'action. Sur la toile de fond des techniques de consultation populaire, l'usage de la violence perd finalement toute crédibilité normative. Il apparaît comme doublement condamnable : en tant que solution moralement réprouvée et en tant que moyen de pression politiquement inefficace. Avec la généralisation de la pratique du vote, des procédures nouvelles existent qui doivent permettre à chacun de manifester son désaccord. Conçu en termes d'"opinion électorale" et à partir du postulat de l'égalité numérique des voix, l'expression du mécontentement social peut disposer d'un espace propre, spécialement réservé à cet effet : celui que délimitent les frontières de la salle de vote et auquel le temps a conféré désormais le visage solennel d'un sanctuaire civique.

Revenir à la partie précédente

\section{NOTES}

1. Tilly (C., L., R.), The Rebellious Century, 1830-1960, Londres, J.M.Dent \& Sons, 1975, pp.62-68.

2. Cf. Braud (P.), Le jardin des délices démocratiques, Pour une lecture psycho-affective des régimes pluralistes, Paris, Presses de la .F.N.S.P., 1991, p.141 sq.

3. Cf. Tilly (C.), La France conteste de 1600 à nos jours, Paris, Fayard, Coll. L'espace du politique, 1986, notamment pp.541-551 et Favre (P.) dir., La manifestation, Paris, P.F.N.S.P., 1990.

4. Archives de la Préfecture de Police (A.P.Po), carton Ba 222, note manuscrite sans date.

5. A.P.Po, carton Ba 220, Le Téléphone, édition du 28 avril 1902.

6. A.P.Po, carton Ba 238, Le Radical, édition du 25 avril 1910. 
7. Un parallèle peut être établi ici avec la répression des attitudes passionnelles que traduisent certains usages considérés comme illégitimes du bulletin de vote ( cf. Déloye (Y.), Ihl (O.), "Des voix pas comme les autres. Votes blancs et votes nuls aux élections législatives de 1881", Revue française de science politique, vol. 41, n², avril 1991, p.167 sq). Si ces derniers sont annulés, les comportements déviants sont à leur tour réprimés au nom d'une conception impersonnelle et uniforme de l'acte du vote.

8. A.P.Po, carton Ba 222, Rapport de police en date du 12 mai 1902.

9. A.P.Po, carton Ba 222, Dépêche télégraphique en date du 27 avril 1902.

10. A.P.Po, carton Ba 238, Dépêche télégraphique en date du 24 avril 1910.

11. A.P.Po, carton Ba 222, Rapport de police en date du 27 avril 1902.

12. Idem. De telles rixes se déroulèrent également pendant la campagne électorale (rapports de police du 21 et du 22 avril 1902).

13. Sur ce point, voir Garrigou (A.), Le vote et la vertu, Comment les français sont devenus électeurs, Paris, Presses de la F.N.S.P., 1992, p.109 et sq.

14. A.P.Po, carton Ba 260, Dépêche télégraphique en date du 8 mai 1904

INDEX

Mots-clés : démocratie, histoire, sociologie historique 